\title{
Coupled climate and soil arsenic stress increase global warming potential from paddy soil
}

\author{
E. MARIE MUEHE ${ }^{1}$, ALANDRA LOPEZ ${ }^{2}$ AND SCOTT \\ FENDORF $^{2}$ \\ ${ }^{1}$ Helmholtz Center for Environmental Science UFZ \\ ${ }^{2}$ Stanford University \\ Presenting Author: eva-marie.muehe@uni-tuebingen.de
}

Almost $5 \%$ of global anthropogenically derived greenhouse gas emissions originate from agricultural practices. Rice paddies contribute the majority of methane and nitrous oxide emissions, which have 25 and 300 times, respectively, the warming effect of carbon dioxide on a per molecule basis. Greenhouse gases are microbially produced and consumed in the soil. The interplay and balance of these microorganisms explicitly determines whether or not greenhouse gases are released from soils into the atmosphere. The presence, abundance, diversity and activity of the microbial community in the soil is easily altered by soil contamination. The most relevant soil contaminant in rice paddies is arsenic. Its bioavailability and redox speciation was shown to tightly link to climatic change. Thus, with an increasing demand for rice, it is important to understand whether and to what extent greenhouse gas emissions from rice paddies will be altered in the future with increasing soil arsenic concentrations.

We performed soil incubations under today's climate and the most likely climate scenario of the year 2100, with doubled atmospheric carbon dioxide concentrations and a five-degree Celsius increase in temperature (postulated by the Intergovernmental Panel on Climate Change). We find that a shift in climatic conditions increased the partitioning of arsenic from solids to solution in Californian and Bangladeshi paddy soil, which is supported by an increase in the abundance of iron(III)- and arsenic(V)-reducing microorganisms. Climatedriven increases in arsenic bioavailability in the soil resulted in an increased global warming potential from both paddy soils. While nitrous oxide was the main greenhouse gas emitted from the Bangladeshi soil, methane dominated emissions from the Californian soil. To explain the observed differences in greenhouse gas emissions, we will provide budgets for waterextractable and solid phase carbon and nitrogen, and correlate them to $16 \mathrm{~S}$ rRNA gene sequencing datasets and gene and transcript copy numbers specific to the cycling of nitrogen and methane in the soil.

Our study highlights the importance of soil contamination as an additional parameter affecting future greenhouse gas emissions from agricultural soils. It further provides a thorough understanding of the key biogeochemical processes altered by climate and contamination in rice paddies. 